\title{
MORTALIDAD PERINATAL ESTUDIO COLABORATIVO INSTITUCIONAL HOSPITALES DEL SUR DEL PERÚ. 2000
}

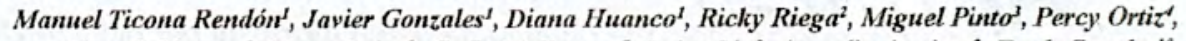
César Solórzanos, Marta Bejar, Sofia Mollapaza ${ }^{7}$, Luisa Lius, Aucadio Aguirres, Fredy Segales ${ }^{20}$, Elert Zapata ${ }^{11}$, Ludgardo Astorga ${ }^{12}$, Andrea Rondón ${ }^{13}$, Federico Manrique ${ }^{44}$, Jorge Aldazabal ${ }^{15}$.
\end{abstract}

\section{RESUMEN}

La mortalidad perinatal da una imagen del nivel de desarrollo y calidad de salud de los pueblos, además permite medir el estado de salud del producto de la concepción durante los últimos meses de vida intrauterina y los primeros 6 días de vida extrauterina. Es la primera causa de mortalidad infantil, se requiere conocer mejor nuestra realidad para elaborar estrategias que permitan superar esta situación.

El objetivo es conocer la magnitud de la mortalidad perinatal en 15 hospitales representativos del sur del Perú y sus componentes fetal tardío y neonatal precoz y las causas relacionadas con este período; además, compararlas según altitud, institución de salud, estratos de pobreza, número de partos y control prenatal

Estudio prospectivo, descriptivo y cooperativo sobre mortalidad perinatal hospitalaria, durante el año 2000, usando el Sistema Informático Perinatal en hospitales del MINSA y el Sistema de Vigilancia Perinatal en hospitales de EsSalud.

Se encontró un total de 25940 nacimientos, de los cuales 690 fueron muertes perinatales, 367 óbitos fetales tardíos y 323 neonatales precoces. La tasa de mortalidad perinatal hospitalaria en el sur del Perú es de 23,46 por mil nacimientos, siendo sus componentes fetal tardío 13,76 por mil nacimientos y neonatal precoz 9,70 por mil nacidos vivos. La relación de mortalidad fetal tardía y neonatal precoz fue de 1.5 a 1 . El examen anatomopatológico de muertes neonatales solamente se realizó en tres hospitales y sólo en uno las muertes fetales.

Las principales causas de mortalidad neonatal precoz fueron: dificultad respiratoria (35,9\%), infecciones (25,2\%), encefalopatía hipóxico isquémica $(17,6 \%)$ y malformaciones congénitas $(12,8 \%)$. Las principales causas específicas fueron membrana hialina $(30,3 \%)$ y septicemia $(24,1 \%)$. El $57,8 \%$ de las muertes neonatales precoces ocurrieron en las primeras 48 horas de vida.

Las principales causas de mortalidad fetal tardía fueron: otras afecciones y las mal definidas (41,3\%), complicaciones de la placenta cordón umbilical o membranas (14,2\%), hipoxia intrauterina y asfixia $(14,2 \%)$, malformaciones congénitas $(7,8 \%)$ y hemorragias $(7,3 \%)$.

Los hospitales que presentaron mayor mortalidad perinatal fueron: Carlos Monge de Juliaca, Santa Rosa de Puerto Maldonado, Lorena y Regional del Cusco y Núñez Butrón de Puno, todos del Ministerio de Salud (MINSA), siendo la posibilidad de morir el doble que en hospitales de EsSalud.

Concluimos que la tasa de mortalidad perinatal y sus componentes fetal tardío y neonatal precoz de los hospitales representativos del sur del Perú, se encuentra en el promedio de estudios nacionales y guarda estrecha relación con la altitud del lugar donde se ubican los hospitales estudiados, con el nivel de pobreza de la población, con la institución de salud a la que pertenece y tiene una relación inversa con el porcentaje de controles prenatales.

1.Dr. del Hosp. H.Unanue Tacna, 2.Dr. EsSalud Tacna, 3.Dr. EsSalud Arequipa, 4.Dr. H.Delgado Areq., S. Dr: Goyeneche Areq., 6. Dra.M.Núñez Puno, 7. Dra. C.Monge Juliaca, 8. Dra. EsSalud Ilo, 9. Dr. Refe.MINSA Ilo, 10. Dr. EsSalud Moqueg, 11. Dr. Refer. MINSA Moqueg., 12. Dr. Regional Cusco, 13. Dra. Lorena Cusco, 14. Dr. EsSalud Cusco, 15. Dr. Sta.Rosa Pto.Mald. 


\section{ABSTRACT}

The Mortality Perinatal gives an image of the development level and quality of health of the towns, it also allows to measure the state of health of the product of the conception during the last months of intra-uterine life and the first 6 days of life extrauterina. It is the first cause of infantile mortality, it is required to know our reality better to elaborate strategies that allow to overcome this situation.

The objective is to know the magnitude of the mortality perinatal in 15 representative Hospitals of the South of the Peru and its late fetal components and precocious neonatal and the causes related with this period; also to compare them according to altitude, institution of health, strata of poverty, number of childbirths and prenatal control

I study prospective, descriptive and cooperative about mortality hospital perinatal, during the year 2000, using the Computer System Perinatal in hospitals of the MINSA and the System of Surveillance Perinatal in hospitals of EsSalud.

He/she was a total of 25,940 births, of which 690 were deaths perinatales, 367 late fetal deaths and 323 precocious neonatales. The rate of mortality hospital perinatal in the south of the Peru is of 23.46 for a thousand births, being its late fetal components 13.76 for a thousand births and precocious neonatal 9.70 for thousand born alive. The relationship of late fetal mortality and precocious neonatal went from 1.5 to 1 . The exam anatomopatológico of deaths neonatales, was only carried out in three hospitals and only in one in the fetal deaths.

The main causes of mortality precocious neonatal were, breathing Difficulty (35.9\%), Infections (25.2\%), encefalopatía hipóxico isquémica (17.6\%) and congenital malformaciones (12.8\%). The main specific causes were membrane hialina $(30.3 \%)$ and septicemia $(24.1 \%) .57 .8 \%$ of the deaths precocious neonatales happened in the first 48 hours of life.

The main causes of late fetal mortality were: other affections and the not well defined ones $(41.3 \%)$, complications of the placenta umbilical cord or membranes (14.2\%), intra-uterine hipoxia and it suffocates (14.2\%), congerital malformaciones (7.8\%) and you hemorrhage (7.3\%).

The hospitals that presented bigger mortality perinatal were: Carlos Monge of Juliaca, Santa Rosa of Port Maldonado, Lorena and Regional of the Cusco and Núnez Butrón of Fist, all of the Ministry of Health (MINSA), being the possibility to die twice as much that in hospitals of EsSalud.

We conclude that the rate of mortality perinatal and their late fetal components and precocious neonatal of the representative hospitals of the South of the Peru, it is in the average of national studies and they keep narrow relationship with the altitude of the place where the studied hospitals are located, with the level of the population's poverty, with the institution of health to the one that belongs and he/she has an inverse relationship with the percentage of prenatal controls.

\section{INTRODUCCIÓN}

El concepto de Perinatología surge como una idea integradora orientada a defender el producto de la concepción en el momento de máxima vuinerabilidad y asi proteger el futuro de la humanidad (CLAP-OPS/ OMS).

Durante el período perinatal, el producto de la concepción está sometido a una serie de riesgos que dependen fundamentalmente del ambiente materno en el cual crece y se desarrolla, y del ambiente exterior. Las afecciones perinatales que no logran producir la muerte, ocasionan con frecuencia secuelas neurológicas y motoras que llegan a constituir una pesada carga para la familia y la sociedad.

El conocimiento de la mortalidad perinatal es un hecho de gran importancia porque nos da una imagen del nivel de desarrollo y la calidad de salud de los pueblos; además, nos permite medir el estado de salud del producto de la concepción durante los últimos meses de vida intrauterina y los primeros 6 días de vida extrauterina. 
La OMS informa que la mortalidad ligada al embarazo y parto constituye más de la mitad de la mortalidad infantil; así, las afecciones originadas en el período perinatal representan el $55 \%$ de la mortalidad infantil en EEUU; $35,8 \%$ en Chile; $55 \%$ en Argentina; $39,2 \%$ Colombia; $29,8 \%$ en México y $56 \%$ en el Perú.

En el mundo desarrollado, las tasas de mortalidad perinatal inician un descenso gradual, con la mejoría de las condiciones de salud, un mejor entendimiento de la fisiología reproductiva, el trabajo interdisciplinario en equipo; y los avances tecnológicos en el estudio del binomio madre-niño, como la ultrasonografia, la amniocentesis, fetoscopia, el doppler, la detección antenatal de trastornos fetales y de malformaciones, el tratamiento oportuno al nacimiento de alteraciones diagnosticadas in útero, los primeros pasos de la cirugía fetal, la increíble sobrevida de neonatos muy pequeños.

Como ha sucedido en otros paises desarrollados, e incluso latinoamericanos que han logrado avances importantes en el área de salud materno infantil (Cuba, Costa Rica y Chile); en nuestro pais la mortalidad perinatal es la primera causa de muerte en la población infantil; esto sucede ya que se ha logrado buen desarrollo a nivel nacional de los diversos programas, como el de control de EDA, IRA, del PAl y el de Planificación Familiar.

En el presente siglo, las tasas de mortalidad perinatal en los paises desarrollados han disminuido en forma acelerada, alcanzando cifras tan bajas como 7,3 en Suiza, 7,9 Alemania, 8,6 Suecia, 8,7 Japón y 10,8 EEUU. Mientras que en los países de América Latina las tendencias son diferentes, con rango de 15 a 95 , encontrándose el Perú en el promedio regional de 35 .

En nuestro país se han realizado dos estudios nacionales: Cervantes, Watanabe y Denegri (1985) en 123 hospitales del MINSA y EsSalud encuentran Tasa de Mortalidad Perinatal (TMP) hospitalaria de 19,1 ; y Villanueva, Lee y García de los Ríos, en Hospitales de EsSalud (1998), una tasa de 20,2.

No habiendo estudió previo de mortalidad perinatal en la región sur del pais, consideramos importante conocer la salud perinatal realizando un estudio en hospitales representativos, donde se atienden más de la mitad de los nacimientos, por lo cual dicha información contribuirá de manera sustancial al conocimiento de las características y dinámica de la mortalidad perinatal en esta región del país.

\section{MATERIAL Y MÉTODOS}

\section{Objetivos}

\section{a) Objetivo General}

Conocer la magnitud de la mortalidad perinatal en hospitales del sur del Perú, indicador importante de la Salud Perinatal Regional.

\section{b) Objetivos Especificos}

- Cuantificar la mortalidad perinatal hospitalaria del sur del Perú.

- Identificar las principales causas de la mortalidad perinatal y sus componentes fetal tardio y neonatal precoz.

- Comparar las tasas de mortalidad perinatal según regiones geográficas, institución de salud, estratos de pobreza, número de partos y control prenatal.

\section{Tipo de Investigación}

Estudio epidemiológico, descriptivo, prospectivo y cooperativo sobre mortalidad perinatal institucional en 15 hospitales representativos del Sur del Perú.

\section{Recolección y procesamiento de la información:}

Se escogió el período del 1 de enero al 31 de diciembre del 2000 , durante el cual se atendió en los hospitales del sur del Perú un total de 25940 nacimientos, de los cuales 690 fueron muertes perinatales, lo que representa el $100 \%$ de las muertes ocurridas en este periodo.

El instrumento que se utilizó fue la Historia Clínica Perinatal del CLAP (OPS/OMS) y se recolectó la información de los libros de registro de los Servicios de Obstetricia y Neonatología, así como del Sistema Informático Perinatal en hospitales del MINSA y del Sistema de Vigilancia Perinatal en hospitales de EsSalud.

Los casos del estudio fueron procesados en el Programa EPIINFO (V.6). 


\section{Instituciones Participantes}

Participaron en el estudio 15 hospitales del sur del Perú, con la coordinación general del Dr. Manuel Ticona:

- Hospital Hipólito Unanue de Tacna del MINSA

- Hospital de EsSalud de Tacna

- Hospital Nacional del Sur de EsSalud de Arequipa

- Hospital Honorio Delgado de Arequipa del MINSA

- Hospital Goyeneche de Arequipa del MINSA

- Hospital Manuel Núñez Butrón de Puno del MINSA

- Hospital Carlos Monge Medrano de Juliaca del MINSA

- Hospital de EsSalud de llo

- Hospital Referencial del MINSA llo

- Hospital de EsSalud de Moquegua

- Hospital Referencial del MINSA Moquegua

- Hospital Regional del Cusco del MINSA

- Hospital Antonio Lorena del Cusco del MINSA

- Hospital de EsSalud del Cusco

- Hospital Santa Rosa de Puerto Maldonado del MINSA

\section{RESULTADOS}

Durante el año 2000 ocurrieron en los 15 hospitales de estudio 25940 nacimientos, de los cuales fueron nacidos vivos 25573 y 367 natimuertos.

El mayor número de nacimientos ocurrieron en los hospitales Honorio Delgado de Arequipa con 5326, Regional del Cusco 2956 e Hipólito Unanue de Tacna 2950. Y los menores en los hospitales de EsSalud de Ilo 267 y EsSalud de Moquegua 360.

Ocurrieron 690 muertes perinatales, de las cuales 367 fueron muertes fetales tardias y 323 muertes neonatales precoces.

La menor tasa de mortalidad perinatal encontrada fue en el Hospital de EsSalud de llo con 7,4 x 1000 n.v. y la más alta en el hospital del MINSA de Juliaca con 55,9 ; siendo la tasa general de 23,46 por mil nacimientos.

La tasa general de mortalidad fetal tardia fue 13,76 por mil nacimientos, oscilando entre 6,04 en el Hospital Nacional del Sur EsSalud de Arequipa y 24,4 en Hospital Núñez Butrón del MINSA Puno.
La tasa general de mortalidad neonatal precoz fue 9,70 por mil nacidos vivos, con un rango de 0 en el Hospital de EsSalud de llo y 35 en el Hospital Carlos Monge de Juliaca.

En la mayoria de hospitales, no se realizó el estudio anatomopatológico de los fallecidos, sobre todo en muertes fetales.

La mortalidad fetal tardía fue menor que la mortalidad neonatal precoz en los hospitales de EsSalud de Arequipa y MINSA Juliaca; esta relación fue de 1 a 1 en los hospitales de EsSalud Moquegua, Honorio Delgado de Arequipa, Lorena y Regional del Cusco y fue mayor en el Hipólito Unanue y EsSalud de Tacna, MINSA Moquegua e Ilo, Goyeneche Arequipa, EsSalud Cusco, Núñez Butrón de Puno y Santa Rosa de Puerto Maldonado. (Gráfico No 1).

Las principales causas de muerte neonatal precoz fueron: sindrome de dificultad respiratoria $(35,9 \%)$, siendo la más importante la enfermedad de Membrana Hialina con $30,3 \%$ del total; en segundo lugar las infecciones $(25,2 \%)$, la septicemia fue la causa más importante con $24,1 \%$ del total, en tercer lugar la Encefalopatía Hipóxico isquémica $(17,6 \%)$, luego las Malformaciones Congénitas $(12,8 \%)$ y finalmente la inmadurez $(5,9 \%)$. (Gráfico $\mathrm{N}^{\circ} 2$ ).

La membrana hialina constituye la principal causa específica de mortalidad con una tasa regional de 3,8 por mil n.v., siendo mayor en el Hospital Regional del Cusco con una tasa de mortalidad de 6,9, en Juliaca 6,7 y Puerto Maldonado 6,5. (Gráfico $\mathrm{N}^{\circ} 3$ ).

La segunda causa específica de mortalidad fue la septicemia con una tasa regional de 3,1 por mil n.v., siendo mayor en Juliaca con una tasa de 20,2 , Lorena y Regional del Cusco con 5,6 y 3,8 respectivamente. (Gráfico $\mathrm{N}^{\circ} 4$ ).

La tercera causa de muerte fue la encefalopatía hipóxico isquémica con una tasa de mortalidad de 2,2 por mil n.v., siendo mayor en Juliaca con una tasa de 8,8 , Regional del Cusco 4,1 y EsSalud de Moquegua 2,8 por mil nacimientos. (Gráfico $\mathrm{N}^{0} 5$ ).

En relación al tiempo de vida de los recién nacidos fallecidos en la primera semana, encontramos que el $7,6 \%$ tuvieron menos de 1 hora de vida, $35 \%$ de una hora a menos de 24 horas, $15,2 \%$ de 24 a menos de 48 horas y el $42,2 \%$ más de 48 horas de vida. (Gráfico $N^{\circ} 6$ ).

Las causas más frecuentes de muerte fetal tardia fueron otras afecciones y las mal definidas $(41,3 \%)$, 
complicaciones de la placenta, cordón umbilical o membranas e hipoxia intrauterina y asfixia $(14,2 \%)$, luego malformaciones congénitas $(7,8 \%)$ y hemorragias $(7,3 \%)$. (Gráfico $\left.N^{0} 7\right)$.

La tasa de mortalidad perinatal y sus componentes guardan estrecha relación con la altitud sobre el nivel del mar, a mayor altitud mayor mortalidad, como ocurre en los hospitales de Puno y Juliaca, que a su vez es semejante al del Hospital de Puerto Maldonado (Gráfico № 8).

La tasa de mortalidad perinatal y sus componentes guardan estrecha relación con la institución prestadora de salud, las tasas de mortalidad son e! doble en hospitales del Ministerio de Salud en relación a hospitales de EsSalud (Gráfico No 9 ).

La tasa de mortalidad perinatal y sus componentes guardan relación directa con el estrato socioeconómico de las gestantes; así, a mayor pobreza, mayor mortalidad (Gráfico $N^{\circ} 10$ ). De igual manera con el número de partos (Gráfico $N^{\circ} 11$ ) y una relación inversa con el número de controles prenatales (Gráfico N N 12).

Comparando nuestras tasas de mortalidad perinatal y sus componentes fetal tardía y neonatal precoz en relación con otros estudios nacionales como: Hospitales de EsSalud del Perú (1998) y hospitales de MINSA-EsSalud (1985), se encuentran en el promedio de estudios nacionales (Tabla $\mathrm{N}^{\circ} 11$ ).

\section{DISCUSIÓN Y COMENTARIOS}

A continuación se describen los hechos más importantes, que a juicio de los investigadores han arrojado este proceso: la tasa de mortalidad perinatal refleja en buen grado la realidad de la atención perinatal institucional.

En el presente estudio se han encontrado 25940 nacimientos acontecidos en el año 2000 en 15 hospitales del sur del Perú, hubo 690 productos fallecidos, 367 de los cuales fueron muertes fetales tardías y 323 muertes neonatales precoces; por lo que se desprende que la Tasa de Mortalidad Perinatal (TMP) encontrada en nuestro estudio es de 23,46 por mil nacimientos, y ésta se divide en sus componentes: Tasa de Mortalidad Fetal Tardia (TMFT) con 13,76 por mil nacimientos y Tasa de Mortalidad Neonatal Precoz (TMNP) con 9,70 por mil nacidos vivos, siendo la relación de 1,5 a 1, pero en países desarrollados ésta es de 1 a 1 , es decir, que la posibilidad de morir intraútero es igual que en los primeros 6 dias de vida extrauterina.

Se han realizado dos grandes estudios sobre mortalidad perinatal en el Perú: Cervantes, Watanabe y Denegri (1985) en 123 hospitales del MINSA e IPSS encontraron una TMP de 19,1 (TMFT 9,6; TMNP 9,5 siendo la relación de 1 a 1) y Villanueva, Lee y García (1998) en 35 hospitales de ESSALUD una TMP de 20,2 (TMFT 12,2 y TMNP 8,0, siendo la relación de 1,5 a 1). Podemos concluir que la mortalidad perinatal hospitalaria en el sur del Perú es semejante a los estudios anteriores.

Según la OMS (1998) informa que en América Latina las tendencias de la mortalidad perinatal son diferentes; encontrándose tasas elevadas como 95 en Haití, 55 Bolivia y 45 en Brasil, Ecuador y Guatemala; y bajas como 15 en Chile y Cuba, encontrándose el Perú en el promedio regional de 35. La TMP en nuestro estudio es menor a esta tasa nacional, pero no es comparable ya que la nuestra es hospitalaria.

Los cinco hospitales que presentaron mayor mortalidad perinatal fueron: Carlos Monge de Juliaca, Santa Rosa de Puerto Maldonado, Lorena y Regional del Cusco y Núñez Butrón de Puno, todos del Ministerio de Salud (MINSA) siendo la TMP en hospitales del MINSA el doble en relación con hospitales de EsSalud; esto se debería a múltiples factores, principalmente porque acuden poblaciones de alta marginación y pobreza con niveles de salud deficientes y además no cuentan con adecuada tecnología médica; podemos deducir que el sistema hospitalario del sector público administrado por el Ministerio de Salud es insuficiente para atender a la población materno perinatal; por lo que, si realmente se requiere modificar esta situación, es urgente ampliar y mejorar los servicios de atención materno y perinatal en estos hospitales.

La TMP también guarda estrecha relación con la altitud del lugar donde se ubican los hospitales estudiados, es decir, a mayor altitud mayor mortalidad. Se corrobora la alta mortalidad perinatal en zonas de altura; esta mayor tasa es evidente aun en la ciudad de Arequipa, tanto en el Hospital de EsSalud como en el del MINSA. Comparativamente la TMP en el Hospital de EsSalud de llo (nivel del mar) es de 7,4 por mil, en el de EsSalud de Tacna (500 m.) es de 11,3, en el de EsSalud de Moquegua (1410 m.) de 16,6 por mil y en el de EsSalud de Arequipa $(2350 \mathrm{~m}$.) de 19,6 por mil. Los datos del 
presente estudio muestran que los patrones de salud son diferentes en las poblaciones de la altura que en las de nivel del mar. Existen evidencias para sugerir que los habitantes nativos de la altura no se encuentran completamente adaptados para vivir en dicho ambiente.

La TMP guarda estrecha relación con el estrato socioeconómico de las gestantes; a mayor pobreza, mayor mortalidad perinatal. Asi, en el Mapa de la Pobreza, los departamentos de Puno, Cusco y Madre de Dios se ubican en el estrato IV (pobreza del 70,1$80 \%$ ), donde el riesgo de morir es el doble que en los estratos I ( $31,6-40 \%$ pobreza) y II ( $40,1-50 \%$ pobreza) donde se encuentran los hospitales de Tacna, Moquegua y Arequipa.

La TMP guarda relación con el número de partos que se atiende en estos hospitales, es decir, a mayor número de partos mayor mortalidad, debido a que la atención perinatal es más compleja por el mayor número de embarazos y partos de alto riesgo y mayor referencia de centros de menor complejidad.

La TMP guarda relación con el número de controles prenatales; a mayor porcentaje de controles prenatales, menor mortalidad perinatal, ya que la posibilidad de detectar un factor que signifique riesgo perinatal para controlarlo y evitar daño al producto sólo puede estar dado, lógicamente, bajo un programado y adecuado control durante la gestación.

Se ha realizado una variedad de estudios sobre TMP en diferentes hospitales del Perú, que podemos comparar con la nuestra que es de 23,46 por mil nacimientos. Así, se encuentran tasas menores como las reportadas por Rejas con 11,95 en el Hospital Militar Central, 10,00 en San Bartolomé de Lima y 14,46 en el Rebagliati del IPSS; y mayores a la nuestra como 25,4 en 10 hospitales de Lima y provincias con el Sistema Informático Perinatal (SIP), 32.70 en la Maternidad de Lima, 31,8 en el Alcides Carrión del Callao, 27,0 en el Maria Auxiliadora de Lima y 25,70 en el Hospital Nacional Cayetano Heredia. Podemos concluir que nuestra mortalidad perinatal se encuentra en el promedio a la reportada por la mayoría de estudios nacionales.

La tasa de mortalidad fetal tardía en nuestro estudio fue de 13,76 por mil nacimientos. En otros hospitales de nuestro país se encuentran tasas menores como en el Hospital Alcides Carrión dei Callao con 7,8; en el Militar Central de Lima, Rejas reporta 7,94; Oliveros en el Rebagliati de Lima 7,52 y Urquizo en San Bartolomé, 6,0 . Y mayores a nuestra tasa son reportados por el Hospital Docente de Huacho 14,20; Rodriguez en el Cayetano Heredia de Lima reporta 16,10; en la Maternidad de Lima 19,1; en el estudio de 10 hospitales por el SIP se encontró 18,7; en María Auxiliadora de Lima 21,6; y Chávez en el Hospital de Apoyo III de Sullana con 33,5. Se han realizado dos grandes estudios sobre mortalidad fetal tardia en el Perú: Cervantes y cols. en el año 1985 en hospitales del MINSA e IPSS con 9,6; y Villanueva y cols. en 1998 en hospitales de EsSalud con 12,2 . Podemos concluir que nuestra mortalidad fetal tardia, está en el promedio a la reportada por la mayoria de estudios nacionales.

La Tasa de Mortalidad Neonatal Precoz encontrada fue de 9,70 por mil nacidos vivos. Dentro de las instituciones hospitalarias del pais, encontramos que la TMNP es mayor a la reportada por Rejas en el Hospital Central Militar de Lima con 4,01 Hospital San Bartolomé con 4,0 en el estudio del SIP en 10 hospitales con 6.7 y en el Rebagliati con 6,94; semejante al Hospital Cayetano Heredia con 9,60; y menor a la Maternidad de Lima con 13,60 y al Hospital Alcides Carrión del Callao con 24. Por lo cual concluimos que nuestra TMNP está en el promedio a nivel nacional.

El examen anatomopatológico, que es importante para todo diagnóstico de causa de muerte, solamente se realizó en tres hospitales: MINSA Moquegua $100 \%$, MINSA Tacna $46 \%$ y EsSalud Arequipa en el $14 \%$ de muertes neonatales y solamente se realizaron necropsias en muertès fetales en el hospital MINSA Moquegua. Las necropsias se realizan con más frecuencia en otros estudios como los reportados con Carrasco $55 \%$ en el Rebagliatti, Rejas $71,2 \%$ en el Militar Central y Kobayashi $100 \%$ en la Maternidad de Lima; ejemplos dignos de imitar. La evaluación cuidadosa del óbito perinatal, para diagnosticar la causa de muerte, es importante para una asesoría genética oportuna para la familia.

Las causas más frecuentes de muerte fetal tardía fueron otras afecciones y las mal definidas $(41,3 \%)$, complicaciones de la placenta, cordón umbilical o membranas e hipoxia intrauterina y asfixia $(14,2 \%)$, luego malformaciones congénitas $(7,8 \%)$ y hemorragias $(7,3 \%)$. Al comparar con otros estudios nacionales, Cervantes encuentra la asfixia y malformaciones congénitas como las más importantes de muerte fetal tardia; Chávez en el Hospital de Apoyo III Sullana reporta que las causas de la muerte intraútero fueron las infecciones $(44,6 \%)$, hemorragias $(25 \%)$, hipertensión $(9,8 \%)$ y las mal 
definidas $(16,3 \%)$; Salvador en el hospital Cayetano Heredia reporta a las infecciones como la principal causa $(28 \%)$, seguida de hemorragias $(20 \%)$ y toxemia $(14 \%)$.

$\mathrm{Al}$ analizar las causas de muerte neonatal precoz encontramos como la más importante las enfermedades del aparato respiratorio (35,9\%), dentro de los cuales la enfermedad de membrana hialina alcanza el $30,3 \%$ del total; en segundo lugar las infecciones $(25,2 \%)$ siendo la septicemia la causa más importante con $24,1 \%$ del total; en tercer lugar la encefalopatía hipóxico isquémica $(17,6 \%)$, luego las malformaciones congénitas $(12,8 \%$ ) y finalmente la inmadurez $(5,9 \%)$. Comparando con otros estudios, nuestros resultados son semejantes a los reportados por Rejas en el hospital Central Militar; De la Torre en el hospital Cayetano Heredia reporta como causas más frecuentes la asfixia, malformaciones congénitas e infección; Urquizo en el Hospital San Bartolomé encontró como las causas más importantes las malformaciones congénitas $(26,4 \%)$, membrana hialina $(20,5 \%)$, infecciones $(14 \%)$ y alteraciones matabólico/nutricionales $(11,7 \%)$.

En relación con el tiempo de vida de los recién nacidos fallecidos en la primera semana, encontramos que el $57,8 \%$ ocurrieron antes de cumplir las 48 horas de vida, lo que indica que estas muertes están principalmente relacionadas con factores endógenos, es decir, relacionadas con el embarazo y parto y la atención inmediata del recién nacido. Además demuestra que todavía nuestros hospitales no tienen la tecnología adecuada para resolver problemas de extrema gravedad como inmadurez y membrana hialina y que en algunos casos la referencia es tardia.

\section{CONCLUSIONES}

1. La tasa de mortalidad perinatal hospitalaria en el sur del Perú es de 23,46 por mil nacimientos, siendo sus componentes fetal tardio 13,76 por mil nacimientos y neonatal precoz 9,70 por mil nacidos vivos.

2. La relación de mortalidad fetal tardía y neonatal precoz fue de 1,5 a 1 .

3. El examen anatomopatológico solamente se realizó en tres hospitales: MINSA Moquegua 100\%, MINSA Tacna $46 \%$ y EsSalud Arequipa $14 \%$ de muertes neonatales; y sólo en el hospital del MINSA Moquegua necropsias en las muertes fetales.
4. Las principales causas de mortalidad neonatal precoz fueron, dificultad respiratoria $(35,9 \%)$, infecciones $(25,2 \%)$, encefalopatia hipóxico isquémica $(17,6 \%)$ y malformaciones congénitas $(12,8 \%)$.

5. Las principales causas especificas de mortalidad neonatal precoz fueron membrana hialina $(30,3 \%)$ y septicemia $(24,1 \%)$.

6. El $57,8 \%$ de las muertes neonatales precoces ocurrieron en las primeras 48 horas de vida.

7. Las principales causas de mortalidad fetal tardia fueron: otras afecciones y las mal definidas $(41,3 \%)$, complicaciones de la placenta cordón umbilical o membranas $(14,2 \%)$, hipoxia intrauterina y asfixia $(14,2 \%)$, malformaciones congénitas $(7,8 \%)$ y hemorragias $(7,3 \%)$.

8. Los cinço hospitales que presentaron mayor mortalidad perinatal fueron: Carlos Monge de Juliaca, Santa Rosa de Puerto Maldonado, Lorena y Regional del Cusco y Núñez Butrón de Puno, todos del Ministerio de Salud (MINSA). La posibilidad de morir del perinato en hospitales del Ministerio de Salud es el doble que en hospitales de EsSalud.

9. La TMP guarda estrecha relación con la altitud del lugar donde se ubican los hospitales estudiados, el riesgo de morir del perinato es dos veces mayor si es que nace en altitud que a nivel del mar.

10. La mortalidad perinatal guarda relación directa con el nivel de pobreza de la población, siendo el doble en el estrato IV que en los estratos I y II.

11. El porcentaje de controles prenatales tuvo relación inversa con la mortalidad perinatal, a menor control prenatal mayor mortalidad.

12. La tasa de mortalidad perinatal y sus componentes fetal tardio y neonatal precoz de los hospitales del Sur del Perú se encuentra en el promedio de estudios nacionales.

\section{RECOMENDACIONES}

1. Reconocer y difundir que la etapa perinatal es la de más alto riesgo de la vida, no sólo por la cantidad elevada de muertes, sino además por la alta incidencia de daños graves e irreversibles que se produce en esta etapa.

2. Ampliar la cobertura y mejorar la calidad del control prenatal, porque la mortalidad perinatal 
disminuye al detectar oportunamente las intercurrencias y complicaciones del embarazo y se aplican medidas correctivas, antes de producirse el daño del producto.

3. Mejorar el sistema de vigilancia epidemiológica de mortalidad perinatal, utilizando el enfoque de riesgo para lograr identificar las prioridades y distribución adecuada de los recursos.

4. Aumentar la proporción de necropsias, sobre todo de la mortalidad fetal para determinar la causa básica de muerte e intervenir en ella.

5. Lograr la regionalización de la atención perinatal con el propósito de atender los pacientes de alto

\section{REFERENCIAS BIBLIOGRÁFICAS}

ALVARADO, R.: «Estudio Descriptivo de los Factores Asociados Maternos relacionados a la Mortalidad Perinatal en el Hospital Arzobispo Loayza, 1982-1991». Tesis para optar el grado de Bachiller en Medicina. Universidad Peruana Cayetano Heredia. Lima, 1992.

BARA, M.: «Mortalidad Perinatal: Magnitud del Problema». Niños. (9); Enero 1990.

BECERRA, J.: «Tasa de Mortalidad Asociados a Proceso Originados en el Periodo Perinatal en Estados Unidos, 19861987». Pediatrics Vol 22; (6); Junio 1992.

BERLANGA y Cols: "Morbimortalidad en una Sala de Neonatologia de Segundo Nivel». Bol. Méd. Hosp. Inf. Méx. Vol 47; (1). 1991.

CABRERA, L.: «Muerte Fetal: Incidencia y Causas Asociadas». Tesis para optar Título de Especialista en Ginecologia y Obstetr. Universidad Peruana Cayetano Heredia. Lima, 1990.

CAMPOS, T.: «Mortalidad Infantil no Río de Janeiro, Brasil: áreas de risco e trajetória dos pacientes até os servicos de saúden. Revista Panam. Salud pública. Vol.8, No3. 2000.

CARRASCO, N.: «Muerte Fetab). Revista de la Asociación Peruana de Ginecología y Obstetricia. Lima, Perú. 1991.

CERON, P.: "Causas de Muerte Perinatal en Instituciones de Salud de la Ciudad de México». Bol. Méd. Hosp. Inf. Méx. Vol 48; (11). Nov. 1991.

CERVANTES, R.: «Muerte Materna y Muerte Perinatal en los Hospitales del Perú»). Ministerio de Salud y la Sociedad Peruana de Gineco-Obstetricia. 1988.

CHAVEZ, W.: «Muerte Fetal Intraútero: Factores de Riesgo. Hospital de Apoyo III Sullana». Revista de Obstetricia y Ginecología. Vol.12, № 4. Diciembre 1996.

DE LA TORRE, J.: «Factores de Riesgo Asociados a Muerte Neonatal en Hospital Cayetano Heredia. 1992-1993». Revista de Obstetricia y Ginecología. Vol.41, No1. Enero 1995. riesgo, dotando, a un hospital por departamento, de todos los elementos que les permitan establecer Unidades de Cuidados Intensivos Neonatales y mejorando las referencias y contrarreferencias.

6. Promover la investigación operativa y epidemiológica en el área materno perinatal para identificar problemas prioritarios y proponer soluciones.

7. Finalmente, implementar la iniciativa «Diez Pasos para un Parto Seguro» que constituye una buena estrategia para contribuir a la reducción de la mortalidad perinatal.

DENEGRI, J.: «Mortalidad Perinatal». En: Ludmir A. Ginecología y Obstetricia. Sección I, Capitulo 14, Tema 52. CONCYTEC. Lima, Perú. 1996.

FANOROFF, A.: «Neonatal Perinatal Medicine. Diseases of the Fetus and Infants». 5ta. Edición. Mosby Year Book. 1992.

FOSTER, A.: «The Impact of Prenatal care on Fetal and Neonatal death, 1961-1988». Obstetrics and Gynecology. Vol 79. Jan 1992.

FUENTES, M.: «Factores de Riesgo Asociados a Mortalidad Neonatal, en un hospital de Chiapas, México». Reproducao \& Climaterio. Suplemento 1. Vol. 16. 2001.

GONZALES, G.: «Patrones Demográficos, Reproductivos y de Morbimortalidad en las Poblaciones de Altura del Perú». Acta Andina. Vol 8: 85-93. 1998

HARTLEY, R.: «Perinatal Mortality and Neonatal Morbility rates among twin pairs at different gestational ages: Optimal delivery timing al 37 to 38 weeks gestation». Am J Obstet. Gynecol. February, 2001.

LAZARTE, R.: «Mortalidad Neonatal en el Cusco. 1984-1989». Resúmenes de Il Jornada de Biopatologia Andina. Lima, 1990.

MOZO DE ROSALES, F.: «Morbimortalidad Perinatal del liquido amniótico teñido». Cli.Invest.Gin. Obst. Vol.21, Nº7, 1994.

OLIVEROS, M.: «Análisis de la Mortalidad Perinatal durante dos Décadas en el Hospital Nacional Edgardo Rebagliati Martins, 1970-1990». Diagnóstico. (32); 22-26. 1993.

REJAS, R.: «Mortalidad Perinatal en el Hospital Central Militar 1980-1989». Tesis para optar el grado académico de Bachiller en Medicina. Universidad Nacional de San Agustín. Arequipa, Perú. 1991.

RIVERA: «Morbilidad y Mortalidad en Neonatos de Bajo Peso al Nacer». Bol.Méd.Hosp.Inf.Méx. Vol 48; (2). Feb, 1991.

RODRIGUEZ, E.: «Muerte Fetal y Factores Asociados en el Hospital de Apoyo Cayetano Heredian. Tesis para optar el grado académico de Bachiller en Medicina. Universidad Peruana Cayetano Heredia. Lima, Perú. 1990. 
ROMAN, C.: «Eclampsia. Mortalidad Materna y Perinatal. Hospital Cayetano Heredia. 1991-1997». Revista Peruana de Ginecología y Obstetricia. Vol.45, N4, Octubre 1999.

SALAZAR, M.: «Edad Materna Avanzada como Factor de Riesgo de Morbimortalidad Materna y Perinatal». Revista Peruana de Ginecología y Obstetricia. Vol.45, N02, abril 1999.

SALVADOR, J.: «Muerte Fetal: Incidencia, Causas y Factores de Riesgo. Hospital Nacional Cayetano Heredia, 1992-1993». Revista Peruana de Ginecologia y Obstetricia. Vol.40, No1. Setiembre 1994.

SERPA, L.: «Morbimortalidad Perinatal en el Hospital San Bartolomé, 1998-1999». XXI Congreso Peruano de Pediatría. Boletin Informativo de la Sociedad Peruana de Pediatria. Vol.12, No 1. 2000
TEJADA, R.: «Mortalidad Materna y Perinatal en el distrito de Yauli, Huancavelica 1997». Revista Peruana de Ginecología y Obstetricia. Vol.45, No2, Abril 1999.

TICONA, M.: «Mortalidad Perinatal. Hospital Hipólito Unanue de Tacna 1983-91». El Recién Nacido. Morbi-Mortalidad. Universidad Nacional Jorge Basadre G. de Tacna. 1996.

TICONA, M.: «Factores de Riesgo Asociados a la Mortalidad Perinatal. Experiencia en el Hospital Hipólito Unanue de Tacna. 1992-1997". Revista Peruana de Ginecologia y Obstetricia. Vol.44, N³, Octubre 1998.

URQUIZO, R.: «Mortalidad Perinatal. Experiencia en el Hospital San Bartolomé. Diez años después». Revista Peruana de Ginecologia y Obstetricia. Vol.44, N², Julio 1998.

TABLA 1. TABLA COMPARATIVA DE MORTALIDAD PERINATAL EN HOSPITALES DEL PERU.

\begin{tabular}{|c|c|c|c|c|}
\hline CENTRO HOSPITALARIO & AÑOS & M.F.T. & M.N.P. & T.M.P. \\
\hline Hosp. Hipólito Unanue - Tacna $\left(500^{*}\right)$ & 2000 & 13.90 & 4.48 & 18.31 \\
\hline Hosp. EsSalud - Tacna & 2000 & 9.71 & 1.63 & 11.34 \\
\hline Centro Referencial - Moquegua (1410) & 2000 & 9.13 & 4.61 & 13.64 \\
\hline Hosp. EsSalud - Moquegua & 2000 & 8.24 & 8.33 & 16.57 \\
\hline Centro Referencial - llo (n.m.) & 2000 & 8.20 & 2.70 & 10.90 \\
\hline Hosp. EsSalud - llo & 2000 & 7.41 & 0 & 7.41 \\
\hline Hosp. Goyeneche Arequipa (2350) & 2000 & 19.24 & 5.23 & 24.27 \\
\hline Hosp. Honorio Delgado Areq. (2350) & 2000 & 17.74 & 13.02 & 30.76 \\
\hline Hosp. Nacional EsSalud Areq. (2350) & 2000 & 6.04 & 13.51 & 19.55 \\
\hline Hosp. Nuñez Butrón - Puno (3850) & 2000 & 24.40 & 6.60 & 31.00 \\
\hline Hosp. Carlos Monge-Juliaca $\quad(3850)$ & 2000 & $20.90^{\circ}$ & 35.00 & 55.90 \\
\hline Hosp. Regional - Cusco & 2000 & 14.88 & 16.48 & 31.36 \\
\hline Hosp. Antonio Lorena Cusco $(3400)$ & 2000 & 17.34 & 15.06 & 32.40 \\
\hline Hosp. EsSalud - Cusco & 2000 & 9.00 & 5.90 & 14.90 \\
\hline Hosp. Sta.Rosa Pto.Maldonado (230) & 2000 & 20.20 & 12.90 & 33.10 \\
\hline HOSPITALES DEL SUR DEL PERU & 2000 & 13.76 & 9.70 & 23.46 \\
\hline HOSPITALES DE ESSALUD DEL PERU & 1998 & 12.20 & 8.00 & 20.20 \\
\hline Hosp. María Auxiliadora - Lima & 1995 & 21.60 & 5.40 & 27.00 \\
\hline SIP (10 HOSPITALES) & $88-92$ & 18.70 & 6.70 & 25.40 \\
\hline Hosp. Central Militar de Lima & $80-89$ & 7.94 & 4.01 & 11.95 \\
\hline Hosp. San Bartolomé de Lima & 1999 & 6.00 & 4.00 & 10.00 \\
\hline Hosp. Docente de Huacho & 1995 & 14.20 & & \\
\hline Inst. Nacional Materno Perinatal & 1995 & 19.10 & 13.60 & 32.70 \\
\hline Hosp. Apoyo III Sullana & $94-95$ & 33.50 & & \\
\hline Hosp. Rebagliatti EsSalud - Lima & $92-95$ & 7.52 & 6.94 & 14.46 \\
\hline Hosp. Cayetano Heredia - Lima & 1994 & 16.10 & 9.60 & 25.70 \\
\hline Hosp. Alcides Carrión - Lima & $94-95$ & 7.80 & 24.00 & 31.80 \\
\hline 123 HOSPITALES (MINSA-EsSalud) & 1985 & 9.60 & 9.50 & 19.10 \\
\hline
\end{tabular}



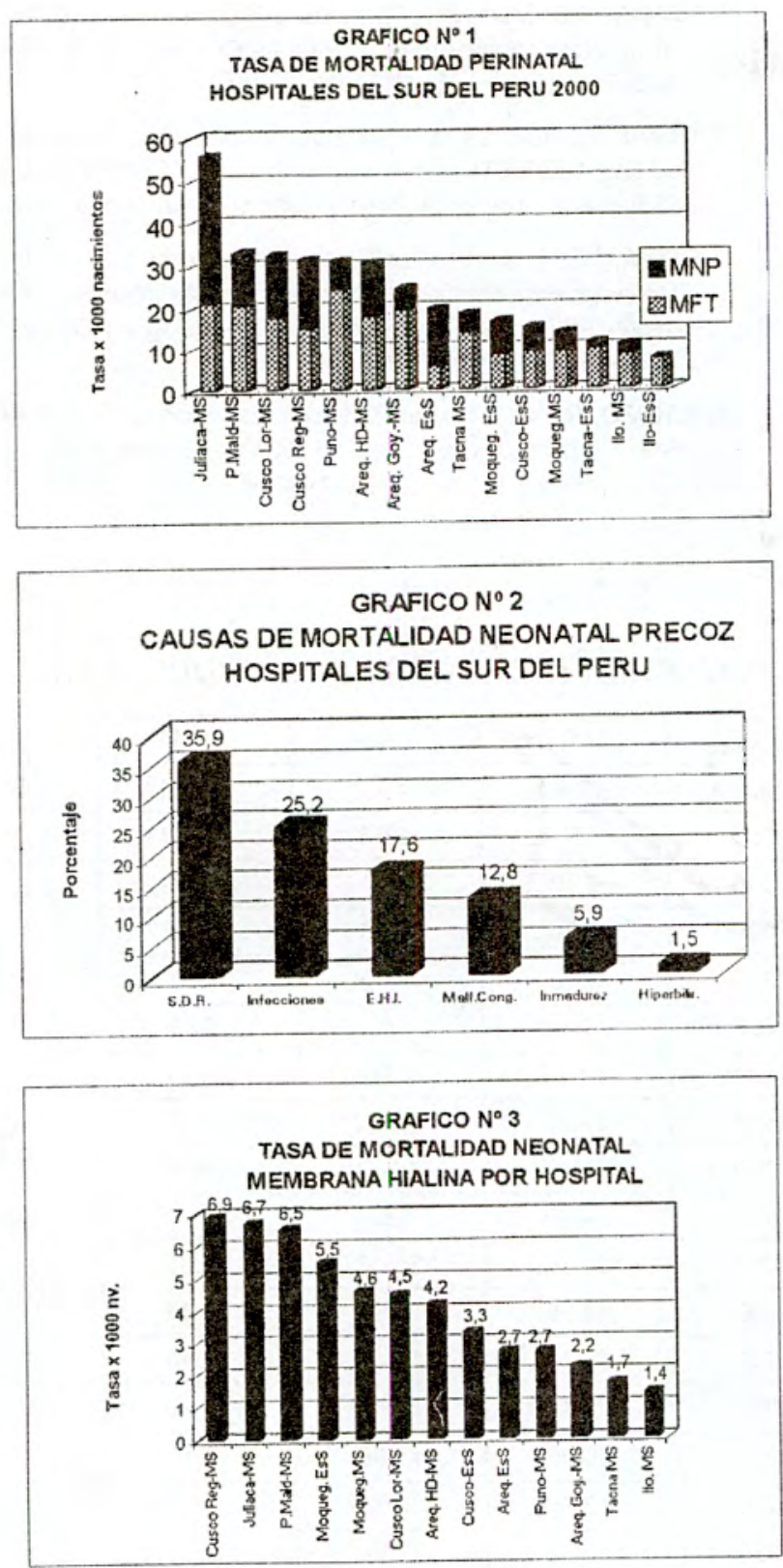
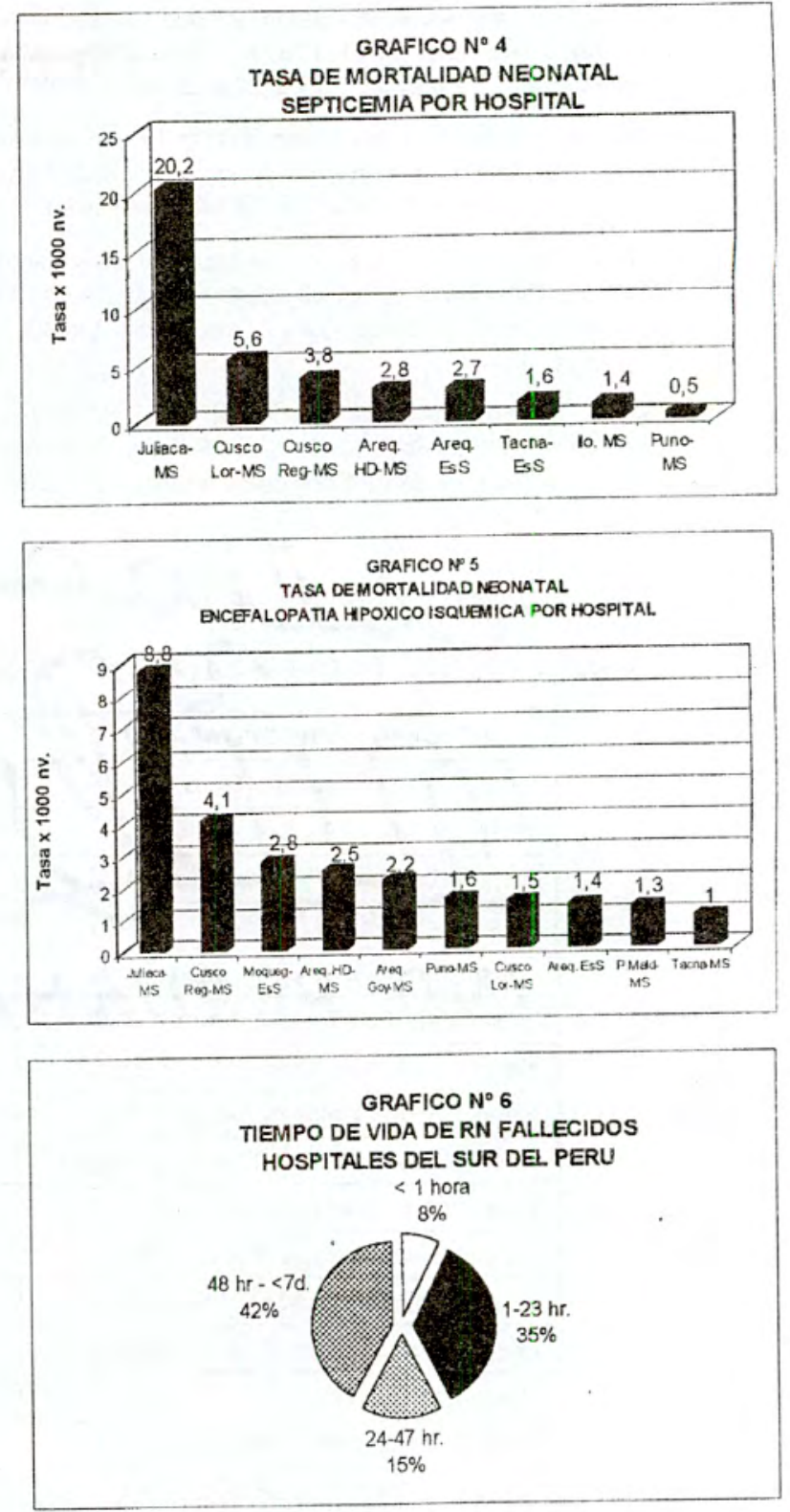

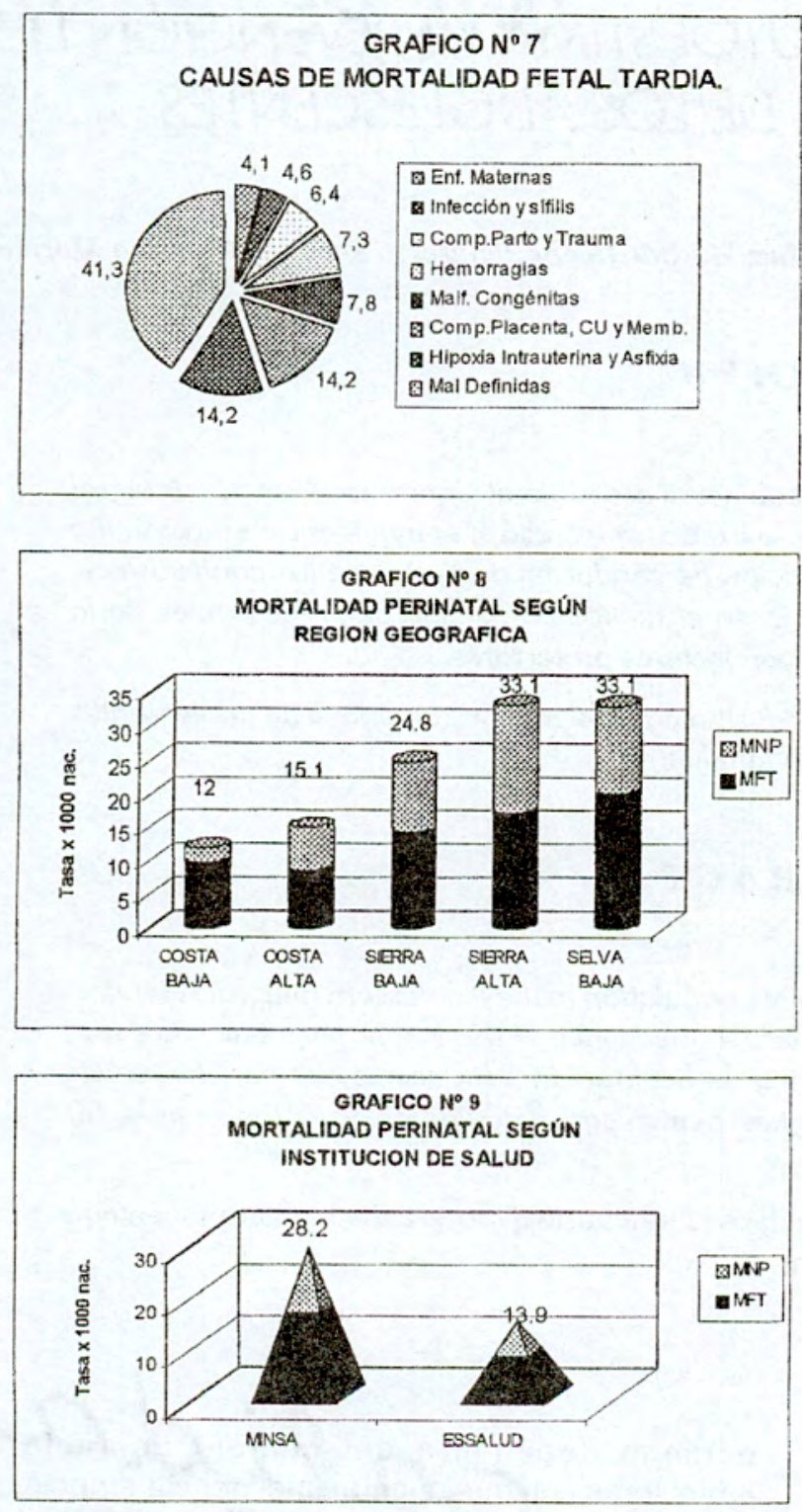
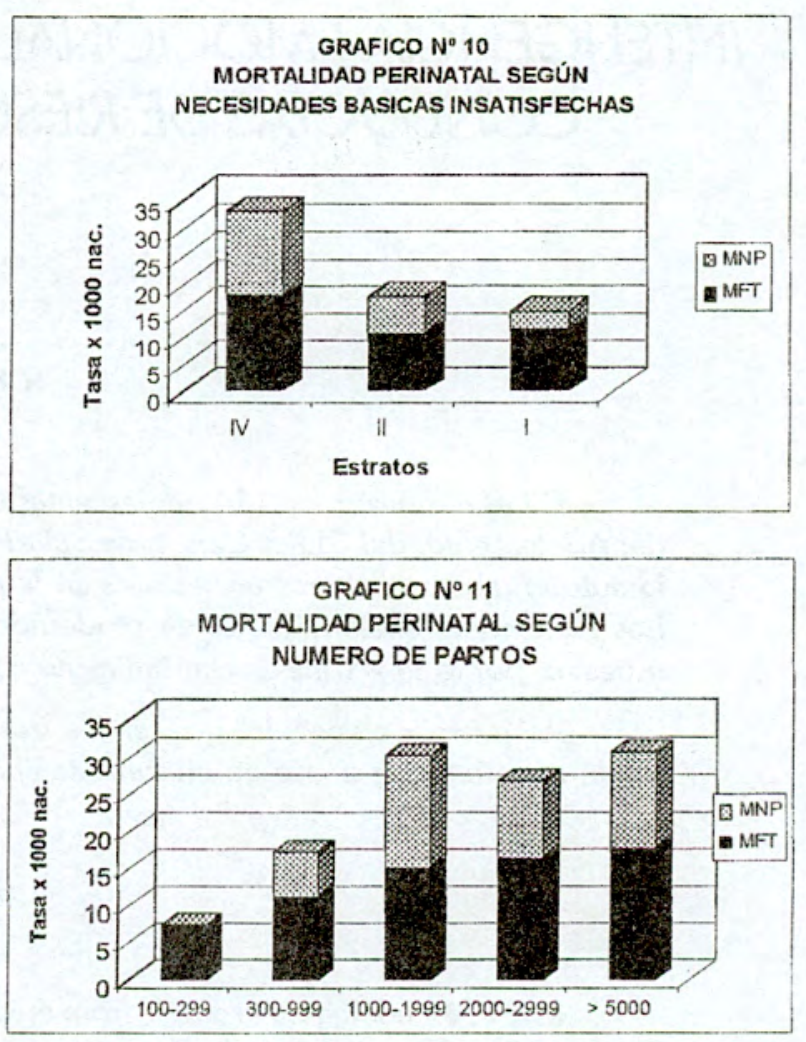

GRAFICO No 12

MORTALIDAD PERINATAL SEGÚN PORCENTANE DE CONTROL PRENATAL.

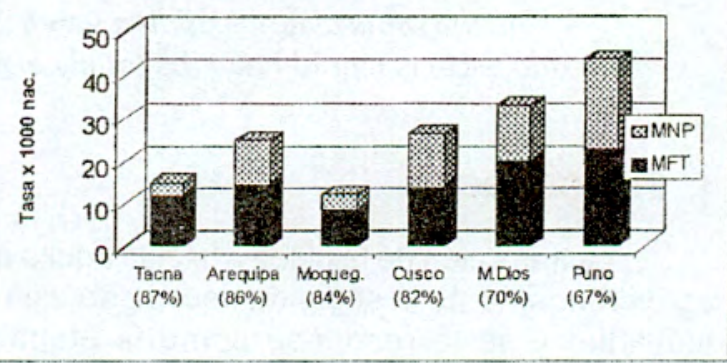

